\title{
Integridad biótica de la microcuenca del Río Chiquito, Morelia, Michoacán, México, basada en la comunidad de macroinvertebrados acuáticos
}

\author{
Miguel Aurelio Piñón Flores ${ }^{1}$, Ricardo Miguel Pérez Munguía ${ }^{2}$, Ulises Torres García ${ }^{3}$ \& \\ Raúl Pineda López ${ }^{4}$ \\ 1. Centro de Referencia Especializado en Bioindicadores, Subdirección de Calidad del Agua, Dirección Técnica del \\ Organismo de Cuenca Balsas de la Comisión Nacional del Agua, México; sefenidae@gmail.com \\ 2. Laboratorio de Entomología "Sócrates Cisneros Paz”, Facultad de Biología, Universidad Michoacana de San Nicolás \\ de Hidalgo, México; pmunguiaricardo@gmail.com \\ 3. Laboratorio de Integridad Biótica, Universidad Autónoma de Querétaro campus aeropuerto, México; \\ ulisestoga@gmail.com \\ 4. Laboratorio de Zoología, Universidad Autónoma de Querétaro, México; rufuspinedal@gmail.com
}

\author{
Recibido 12-XII-2013. Corregido 20-I-2014. Aceptado 13-II-2014.
}

\begin{abstract}
Biotic integrity of the Chiquito River subwatershed, Morelia, Michoacán México, based on the aquatic macroinvertebrate assemblages. The Chiquito River subwatershed supplies $30 \%$ of the potable water consumed by the city of Morelia, Michoacan, Mexico. It is necessary to constantly monitor its waters using metrics that evaluate environmental degradation and aquatic assemblages. In the present study, we used the Index of Biotic Integrity for Aquatic Macroinvertebrate Associations (IBIAMA), the Visual-Based Habitat Assessment, and the Most Probable State to calculate the conservation status of the river at six sites along the main channel, including an upper site as a reference. A total of 51474 specimens were identified, the taxonomic composition was comprised of 69 families distributed into four Phyla, eight classes, eight sub-classes, and 18 orders. The best preserved sites were found in the subwatershed headwater zone. The intermediate zone had impacted hydraulic conditions, environmental quality and biotic integrity. The downstream part of the subwatershed showed the resilient capacity of the river to reestablish macroinvertebrate assemblages and environmental parameters. There is a strong correlation among environmental qualities and the IIBAMA. The use of macroinvertebrates in combination with environmental parameters showed that the Chiquito River subwatershed is stable, rich and integral in aquatic macroinvertebrate assemblages before entering the city of Morelia, and it conserves the processes that regulate the functional dynamics of the subwatershed. Rev. Biol. Trop. 62 (Suppl. 2): 221-231. Epub 2014 April 01.
\end{abstract}

Key words: macroinvertebrate, subwatershed, aquatic assemblages, biotic integrity, resilient.

Los ecosistemas acuáticos mantienen una gran diversidad de organismos, y los impactos como la contaminación inducen cambios en la estructura de la comunidad, la función biológica en el sistema y en los propios organismos, afectando su ciclo de vida, crecimiento y su condición reproductiva (Bartram \& Ballance, 1996; Gibbson, Barbour, Stribling, Gerritsen \& Karr, 1996). Cuando aumenta la degradación ambiental, ocurre la pérdida de taxa sensibles y cambios en la estructura y composición de organismos (Barbour, Gerritsen-Zinder \& Stribling, 1999). Por lo mismo, los estudios dirigidos hacia la descripción y análisis de la estructura trófica y la organización espacial de la comunidad de macroinvertebrados acuáticos debieran ser parte de los programas de monitoreo ambiental en ríos y arroyos.

Con el desarrollo del principio de integridad biótica propuesto por Karr \& Dudley (1981), se desarrollan diversos protocolos, enfocados a la integración de los ensamblajes 
acuáticos como base para la evaluación ambiental (Lyons, 1992). En la elaboración de estas metodologías uno de los requerimientos es que sean capaces de medir alteraciones en los ecosistemas acuáticos, destacando los peces y macroinvertebrados acuáticos, por las diversas respuestas y ventajas que proporcionan (AlbaTercedor \& Sánchez-Ortega, 1988; Barbour et al., 1999). En este sentido, los macroinvertebrados acuáticos son especialmente útiles para reconocer cambios en estos ecosistemas, puesto que constituyen ensamblajes estructurados por una amplia gradación de especies generalistas a microespecialistas que responden rápidamente a los cambios que ocurren en los sistemas acuáticos (Pérez et al., 2006). Bajo la premisa de que la condición física del hábitat es un factor de influencia primaria en la composición y estructura de las comunidades bióticas de los ríos, se ha demostrado que existen correlaciones, entre las comunidades de macroinvertebrados acuáticos con las condiciones físicas y ambientales, que en conjunto responden a las alteraciones dentro de los cauces (Barbour et al., 1999; Merritt, Cummins \& Berg, 2008). Por otro lado, desde el punto de vista hidrológico, si una corriente conserva sus mecanismos de autodepuración, la comunidad de organismos, tienen una composición y estructura que corresponde con sistemas poco alterados; situación que puede ser reconocida usando principios de integridad biótica (Pérez et al., 2006).

El río Chiquito es un sistema acuático de suma importancia para la ciudad de Morelia, capital del estado de Michoacán, México, ya que de acuerdo al plan de manejo propuesto por el Instituto de Investigaciones sobre los Recursos Naturales (INIRENA) y la Facultad de Biología UMSNH (2006), este sistema aporta el $30 \%$ del abastecimiento de agua superficial a la ciudad, además de contener flora y fauna más o menos conservada, como ejemplo, el $25 \%$ de la avifauna registrada en el estado de Michoacán habita en esa zona. De igual forma la sección llamada Loma de Santa María y depresiones aledañas, que se encuentran dentro de la microcuenca del río Chiquito, es denominada como zona de restauración y protección ambiental (PDUCPM, 2010). Este ecosistema, presenta diversos impactos antropogénicos, que van desde cambios en su estructura física hasta descargas rurales y urbanas, que han mermado la calidad del agua, así como la condición de las riberas y sustratos del cauce.

En el presente trabajo se caracterizó la comunidad de macroinvertebrados acuáticos en sitios con distinto grado de perturbación antropogénica. Asimismo, se evaluó la condición geomorfológica describiendo los cambios hidráulicos a través de la microcuenca y de calidad del hábitat en los distintos sitios propuestos. De esta manera se comparó con principios de integridad biótica, los cambios en la composición y estructura funcional de las comunidades de macroinvertebrados acuáticos, asociados a los cambios físicos en sitios con distintos grados de impacto, en la microcuenca del río Chiquito de Morelia Michoacán México.

\section{MATERIALES Y METODOS}

Área de estudio: Haciendo uso de mapas con la hidrología de la microcuenca, los sitios se seleccionaron con base en distintos tipos y grados de impacto ambiental, determinados a partir de recorridos de campo. Se establecieron siete puntos de muestreo, de los cuales seis pertenecen a la microcuenca del río Chiquito, en la cuenca del Río Lerma, identificados como RC-04, RC-05, RC-06, RC-07, RC-08 y San Miguel. El séptimo punto pertenece a la microcuenca del Río Purungueo, en la cuenca del Río Balsas; este último sitio se eligió como un sitio de referencia al presentar poca influencia antrópica y encontrarse en los límites del parteaguas que separa el área de estudio a las dos cuencas, este sitio se identifica como "El Campanario".

Geomorfología: Se caracterizó la geomorfología del cauce de acuerdo al protocolo propuesto por Rosgen (1996), el cual se basa en determinar el estado más probable del cauce al que pertenece el tramo de estudio, complementándolo con la evaluación del substrato propuesto por Bunte \& Abt (2001). La 
estimación de la calidad ambiental se llevó a cabo siguiendo el protocolo para la Valoración de la Calidad Ambiental Visual desarrollado por Barbour et al. (1999), debido a que se trata de un río de montaña se utilizó el criterio de gradiente alto, el cual es usado para cauces con pendientes mayores al 3\%.

Recolecta e identificación: El muestreo se llevó a cabo en época de sequía, por considerarse que las comunidades acuáticas se encuentran más estables (Lyons, 1992; Dall, Friberg, Lindegaard \& Toman, 1995; AFNOR, 2003) y no presentan deriva provocada por los periodos de lluvia. Las muestras se recolectaron siguiendo el Índice de Integridad Biótica para las Asociaciones de Macroinvertebrados Acuáticos "IIBAMA" (Pérez \& Pineda, 2005) utilizando una red acuática tipo "D" de $30 \mathrm{~cm}$ de ancho, con abertura de malla de $300 \mu \mathrm{m}$. Se recolectaron muestras compuestas por ocho submuestras de $0.15 \mathrm{~m}^{2}$, hasta cubrir un área mínima de muestra de $1.20 \mathrm{~m}^{2}$ en la mayor cantidad de hábitats posibles, una por estación. El contenido se preservó en etanol al 80\%, con los datos de recolecta convencionales. El material biológico se separó, identificó y cuantificó en laboratorio utilizando un microscopio estereoscópico, identificando a nivel de familia los organismos encontrados, haciendo uso de los criterios de identificación de Pennak (1989), Wiggins (1994), Thorp \& Covich (2001), Merritt et al. (2008). El reconocimiento del valor de tolerancia, gremio trófico y hábitos propuestos para cada familia, se hizo a partir de Barbour et al. (1999), Mandaville (2002) y Merritt et al. (2008). Los especímenes fueron depositados en la colección del laboratorio de Entomología Sócrates Cisneros Paz, en Morelia Michoacán y en la colección del Centro de Referencia Especializado en Bioindicadores, de la Comisión Nacional del Agua, Cuernavaca, Morelos.

Análisis: Para reconocer la estructura de la comunidad de macroinvertebrados acuáticos, se estimó la riqueza y los índices de diversidad de Shannon y la equidad de Pielou, utilizando el paquete estadístico MSVP v. 3.01 (Kovach Computing Services, 1998). Los análisis correspondientes se hicieron utilizando el nivel taxonómico de familia, puesto que la información sobre los valores de diversidad empleando taxa superiores no ofrece resultados que difieran de manera significativa con la que se obtiene al usar el taxón de especie y en cambio el uso de taxa superiores es más práctico (Williams \& Gaston, 1994; Mitchell, Coan, McFarland \& Lasswell, 1995; Geffrard, Saulnier-Talbot \& Gregory-Eaves, 2011). Para explorar las variables que pueden tener una influencia sobre la estructura funcional de los macroinvertebrados se utilizó el análisis de componentes principales (ACP). La similitud entre los sitios, se estimó con las variables de riqueza y abundancia de los gremios tróficos y hábitos, a partir de un análisis de agrupamiento, utilizando el método de mínima varianza de Ward; ya que ha sido propuesto como un procedimiento aplicable para la mayoría de los problemas de agrupamiento (Székely \& Rizzo, 2005). Estos análisis se realizaron con los paquetes estadísticos, JMP v. 8.0 (SAS Institute 2008) y MVSP v. 3.01 (Kovach Computing Services, 1998). El análisis de integridad se llevó a cabo usando los criterios IIBAMA (Pérez \& Pineda, 2005). Este índice otorga una puntuación de uno a cuatro a cada variable (Cuadro 3), siendo la sumatoria el valor final del índice, que de acuerdo a su puntuación se ubica en una categoría de integridad, pobre, bueno, sub-óptimo y óptimo. Para explicar sobre las relaciones entre el IIBAMA y el Índice de Diversidad de Shannon, con la valoración de la calidad ambiental visual, se hicieron análisis de correlación, utilizando el coeficiente de correlación de Spearman, con el paquete estadístico JMP v. 8.0 (SAS Institute, 2008).

\section{RESULTADOS}

Geomorfología: El tipo de valle que corresponde a la microcuenca del río Chiquito es VI, regulado por los procesos estructurales dominados por fallamiento de tipo normal. Esta clase de valle presenta pendientes menores del 
$4 \%$ y es controlado por el alto confinamiento lateral de los escarpes (encañonamiento). Conforme a la clasificación propuesta por Rosgen (1996), los tipos de cauce que corresponden a estos valles son los de tipo B y al acercarse a la zona de fallas son de tipo $\mathrm{F}$ (Cuadro 1).

Los sitios RC-04, RC-05, RC-06 obtuvieron valores de sinuosidad por debajo de 1.20 que no es lo esperado para el tipo de cauce en este valle, al igual que el sitio RC-08 que además obtuvo una tasa de confinamiento de 2.26 mayor de los 2.20 esperados para la zona. El sitio San Miguel obtuvo la más baja tasa ancho profundidad (5.17) estando fuera del intervalo establecido para este tipo de cauce $(>12)$ (Cuadro 1).

\section{La valoración de la calidad ambiental} visual: Sitúa a los sitios RC-04, RC-05, RC-06 y RC-07 en la categoría de sub-óptimo, los sitios San Miguel y el Campanario en la categoría óptimo, y únicamente el sitio RC-08 que obtuvo la menor calificación con 90 puntos situándose en la categoría marginal (Cuadro 1).
Composición taxonómica: está conformada por cuatro Phyla, ocho clases, ocho subclases, 18 órdenes, 13 subórdenes y 69 familias. Dentro del Phylum Artropoda se encuentran 61 familias que corresponde a el $88.4 \%$ de la riqueza de familias encontradas, de los cuales la clase Insecta posee 59 que equivale al $85.5 \%$ de las riqueza total. Los órdenes Diptera con 15 familias y Trichoptera con 13 fueron los mejor representados con el $21.7 \%$ y $18.8 \%$ respectivamente de las familias encontradas. Se colectaron e identificaron 51474 organismos, siendo los sitios RC-04 y RC-05 donde se encontraron las mayores abundancias con el $27.6 \%$ y $28.6 \%$ respectivamente de la abundancia total, el sitio con menor abundancia fue el RC-08 con el 1.8\%. De las 69 familias reportadas 11 se encontraron en todos los sitios de muestreo. Las familias más abundantes pertenecen a la clase Insecta: Simuliidae 31.6\%, Chironomidae $23.3 \%$ y Baetidae $22.2 \%$.

Estructura trófica: Se encontraron siete gremios tróficos, principalmente conformados por el grupo de los depredadores presentes en

CUADRO 1

Localización geográfica, geomorfología y calidad ambiental en los sitios de muestreo

TABLE 1

Geographic location, geomorphology, and environmental characteristics of sampling sites

\begin{tabular}{|c|c|c|c|c|c|c|c|}
\hline Sitio & Coordenadas & $\begin{array}{l}\text { Altitud } \\
\text { (msnm) }\end{array}$ & $\begin{array}{l}\text { Tipo de } \\
\text { Cauce }\end{array}$ & VCAV & $\begin{array}{l}\text { Categoría } \\
\text { VCAV }\end{array}$ & $\begin{array}{c}\mathrm{D}_{50} \\
(\mathrm{~mm})\end{array}$ & Categoría $\mathrm{D}_{50}$ \\
\hline $\mathrm{RC}-04$ & $\begin{array}{l}19^{\circ} 40^{\prime} 28^{\prime \prime} \mathrm{N} \\
101^{\circ} 9^{\prime} 5 ” \mathrm{~W}\end{array}$ & 1979 Baja & F3 & 116 & Sub-Optimo & $64-128$ & Guijarros pequeños \\
\hline $\mathrm{RC}-05$ & $\begin{array}{l}19^{\circ} 40^{\prime} 27.33 \mathrm{~N} \\
101^{\circ} 8^{\prime} 58.76^{\prime \prime} \mathrm{W}\end{array}$ & 1985 Baja & F4 & 163 & Sub-Optimo & $16-32$ & Grava muy gruesa \\
\hline RC-06 & $\begin{array}{l}19^{\circ} 40^{\prime} 19.26^{\prime \prime} \mathrm{N} \\
101^{\circ} 8^{\prime} 44.58^{\prime \prime} \mathrm{W}\end{array}$ & 1999 Baja & $\mathrm{B} 4 \mathrm{a}$ & 158 & Sub-Optimo & $16-32$ & Grava muy gruesa \\
\hline RC-07 & $\begin{array}{l}19^{\circ} 39^{\prime} 39.98^{\prime \prime} \mathrm{N} \\
101^{\circ} 8^{\prime} 33.62^{\prime \prime} \mathrm{W}\end{array}$ & 2040 Media & $\mathrm{F} 4 \mathrm{~b}$ & 152 & Sub-Optimo & $32-64$ & Grava muy gruesa \\
\hline RC-08 & $\begin{array}{l}19^{\circ} 38^{\prime} 19.59^{\prime \prime} \mathrm{N} \\
101^{\circ} 8^{\prime} 17.68^{\prime \prime} \mathrm{W}\end{array}$ & 2079 Media & E4 & 90 & Marginal & $32-64$ & Grava muy gruesa \\
\hline San Miguel & $\begin{array}{l}19^{\circ} 36^{\prime} 41.65^{\prime \prime} \mathrm{N} \\
101^{\circ} 7 ' 27.91^{\prime \prime} \mathrm{W}\end{array}$ & 2180 Alta & $\mathrm{B} 3 \mathrm{c}$ & 180 & Optimo & $64-128$ & Guijarros pequeños \\
\hline El Campanario & $\begin{array}{l}19^{\circ} 35^{\prime} 44.39^{\prime \prime} \mathrm{N} \\
101^{\circ} 6^{\prime} 42.17^{\prime \prime} \mathrm{W}\end{array}$ & 2075 Alta & B4 & 170 & Optimo & $16-32$ & Grava muy gruesa \\
\hline
\end{tabular}

Valoración de la Calidad Ambiental Visual (VCAV), Diámetro de la partícula \#50( $\left.\mathrm{D}_{50}\right)$ 
todos los sitios y con mayor riqueza, así como los recolectores/colectores. Las menores riquezas las obtuvieron los omnívoros, filtradores/ colectores, cortadores y raspadores. En el sitio RC-07 no se encontraron familias desgarradoras y muy pocas cortadoras (Fig. 1).

Hábito de vida: Se encontraron siete hábitos, la mayor riqueza de taxa son los que viven fijos al substrato del cauce y se encuentran en todos los sitios, excepto en RC-08 donde el hábito nadador tiene la mayor riqueza de taxa. Los trepadores, deslizadores, nadadores y excavadores se encuentran en todos los sitios de muestreo. Los buceadores son exclusivos de los sitios RC-06 y RC-05. La mayor cantidad de taxa deslizadores se encuentran en el sitio el Campanario. El sitio RC-06, es el único donde se ven representados los siete hábitos descritos en este estudio (Fig. 2).

Atributos ecológicos de las comunidades: Las mayores riquezas se encontraron en los sitios RC-04 en la parte más baja de la

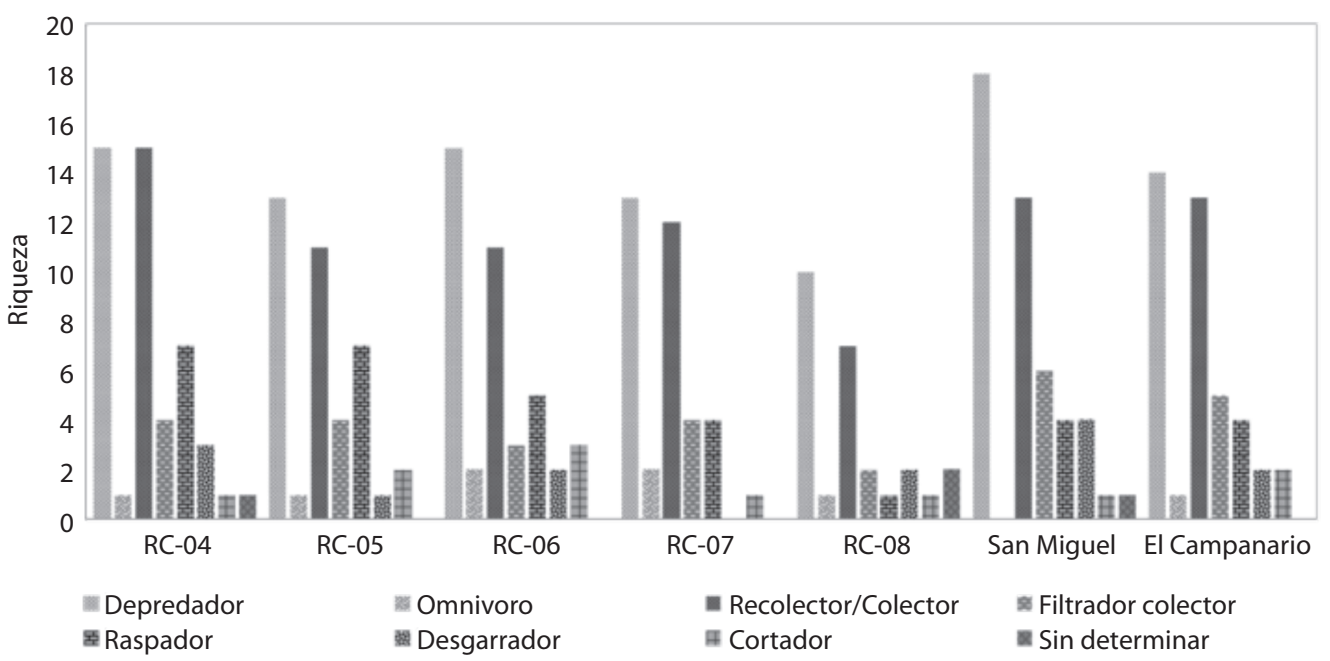

Fig. 1. Riqueza de macroinvertebrados por gremios trófico presentes en los sitios de muestreo.

Fig. 1. Macroinvertebrate richness by trophic guild present at the sampling sites.

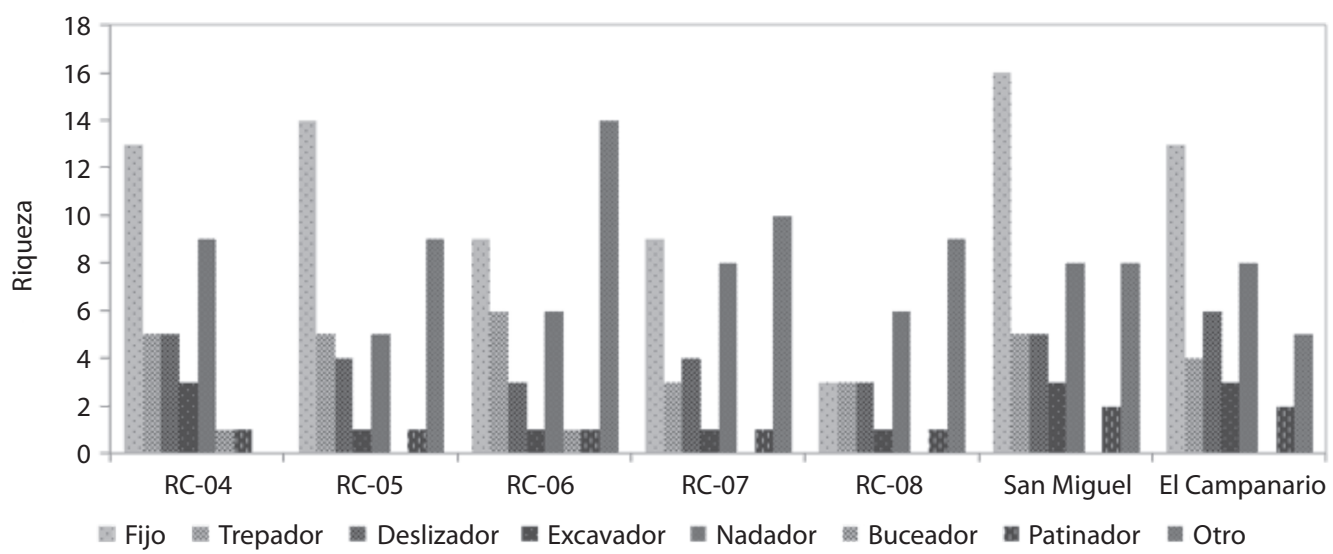

Fig. 2. Riqueza de macroinvertebrados por hábito de vida presentes en los sitios de muestreo.

Fig. 2. Macroinvertebrate richness by life traits present at sampling sites. 
microcuenca y San Miguel siendo el sitio a mayor altura. Sin embargo San Miguel presenta la mayor diversidad con $\mathrm{H}^{\prime}=3.641$, pero no la mayor abundancia $n=2014$. En los sitios RC-04 y RC-05 se encontraron las más altas abundancias, n=14 202 y 14757 respectivamente. El sitio menos abundante fue el RC-08 $\mathrm{n}=937$ y con la menor riqueza $\mathrm{S}=26$ taxa . El sitio menos diverso es el $\mathrm{RC}-07, \mathrm{H}^{\prime}=1.899$, que además presenta una baja equitatividad de la abundancia en las familias encontradas $\mathrm{E}=0.367$ (Cuadro 2).

Estructura de la comunidad: De acuerdo al ACP, se observa que en los dos primeros componentes se explica el $92 \%$ de la variación de los sitios, el primer componente explica el $86.6 \%$ y el segundo el restante $5.4 \%$. Las variables de respuesta explicativas corresponden a la riqueza tanto de hábitos como de gremios tróficos; además de la abundancia de deslizadores, siendo esta la única variable que involucra a la cantidad de organismos. Formando tres grupos bien definidos por su estructura funcional separándose los sitios con mayor riqueza, el Campanario, San Miguel y RC-04; los dos primeros sitios, también corresponden con los de mayor calidad ambiental (VCAV 170 y 180 puntos, respectivamente), en tanto que el tercer sitio de este grupo tiene el valor más bajo de la valoración de la calidad ambiental visual (116), entre los sitios de calidad sub-óptima, al mismo tiempo que corresponde con la parte más baja del área de estudio. Un grupo intermedio se encuentra conformado por RC-06, RC-05 y RC-07 mientras que el sitio RC-08 se excluye siendo el que posee menor riqueza (Fig. 3). De acuerdo al análisis de agrupamiento, se observan dos grupos dados por la riqueza de gremios y hábitos. El primero es integrado por San Miguel, el Campanario y RC-04; el segundo se constituye con el resto de los sitios, es notable que el acomodo de los sitios en este grupo tenga un arreglo que corresponde con los valores de la calidad ambiental, disminuyendo en el sentido de RC-05 a RC-08 (Fig. 4).

Aplicación del IIBAMA: Se utilizaron solo los taxa de los que se tiene información de su tolerancia al impacto. El sitio RC-08 presenta la menor riqueza de taxa y de la combinación de Ephemeroptera, Plecoptera y Trichoptera, con tan solo tres taxa de hábitos fijos lo que le da una calificación de 11 puntos ubicándolo en la categoría de pobre. El sitio RC-07 obtuvo una calificación de 19 puntos con lo que se ubica como un sitio bueno. Mientras que los sitios RC-04, 05 y 06 con 22 puntos calificados como óptimos, siendo San Miguel y el Campanario los que obtuvieron la mejor calificación con 24 puntos, ubicándose en la categoría óptima (Cuadro 3).

De acuerdo al análisis de correlación, se observó que el IIBAMA se encuentra

CUADRO 2

Atributos ecológicos del ensamblaje de macroinvertebrados acuáticos

TABLE 2

Ecological attributes of aquatic macroinvertebrate assemblages

\begin{tabular}{|c|c|c|c|c|}
\hline Sitio & $\mathrm{n}$ & $\mathrm{H}^{\prime}$ & E & S \\
\hline RC-04 & 14202 & 2.614 & 0.471 & 47 \\
\hline RC-05 & 14757 & 2.2 & 0.416 & 39 \\
\hline RC-06 & 3264 & 2.94 & 0.552 & 40 \\
\hline RC-07 & 11902 & 1.899 & 0.367 & 36 \\
\hline RC-08 & 937 & 2.628 & 0.559 & 26 \\
\hline San Miguel & 2014 & 3.641 & 0.655 & 47 \\
\hline El Campanario & 4398 & 2.479 & 0.463 & 41 \\
\hline
\end{tabular}

Abundancia (n), diversidad ( $\left.\mathrm{H}^{\prime}\right)$, equitatividad (E) y riqueza (S). 


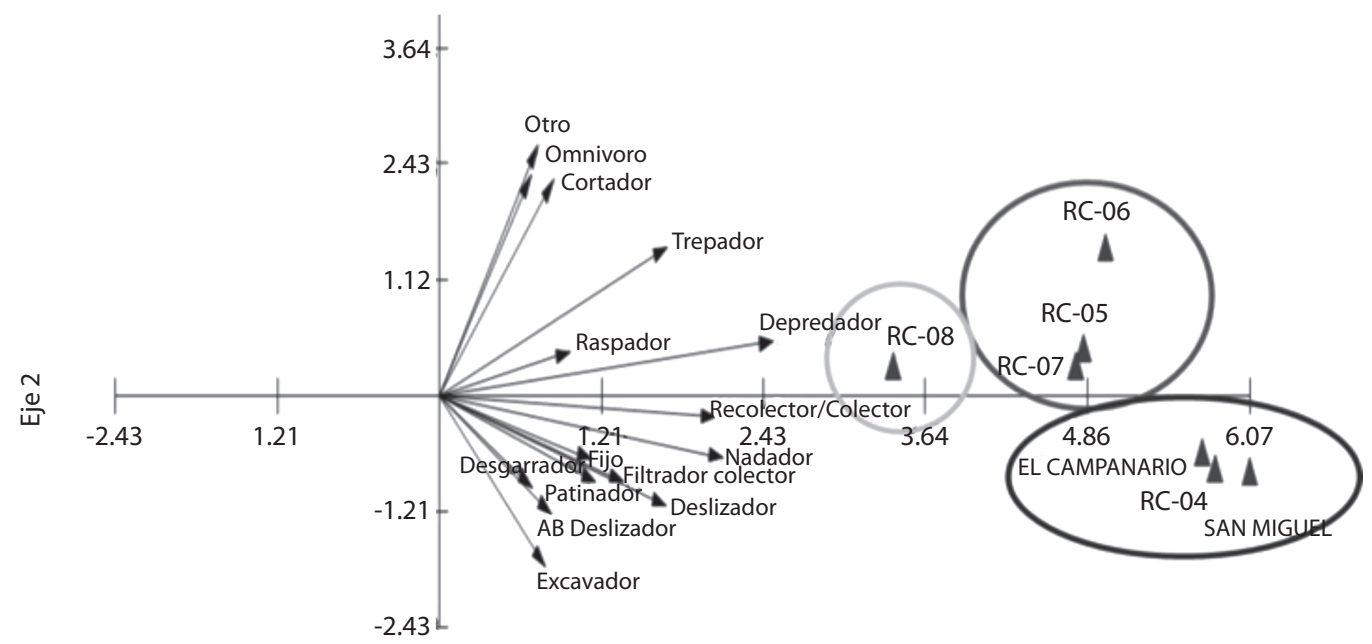

Eje 1

Fig. 3. Análisis de componentes principales utilizando las variables de estructura funcional del ensamblaje de macroinvertebrados acuáticos.

Fig. 3. Principal component analysis using functional structure variables of macroinvertebrate assemblages.

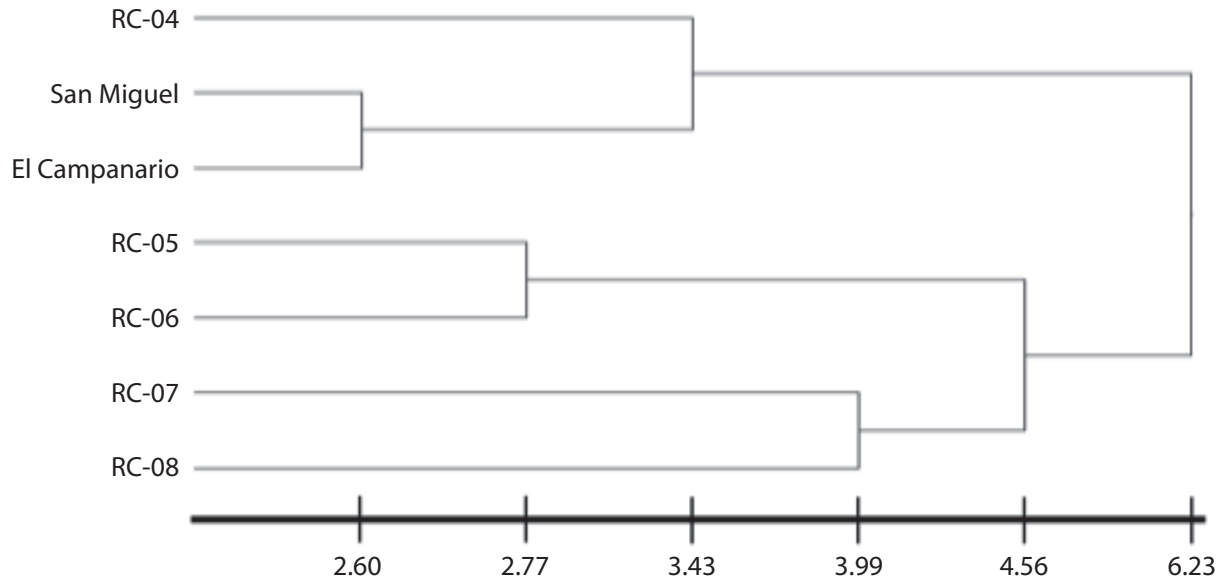

Fig. 4. Índice de similitud de Ward utilizando la riqueza de gremios tróficos y hábitos de vida.

Fig. 4. Ward similarity index using trophic guild richness and life traits.

correlacionado con las variables ambientales de la Valoración de la Calidad Ambiental Visual $(\mathrm{r}=0.82, \mathrm{p}<0.01)$. En tanto que el índice de Diversidad no muestra correlación con la Valoración de la Calidad Ambiental Visual $(\mathrm{r}=0.21, \mathrm{p}=0.70)$.

\section{DISCUSIÓN}

La geomorfología de los sitios corresponde con el tipo de valle esperado a excepción del sitio RC-08 que por su tasa de confinamiento tan baja le confiere características de ríos de planicies o desembocaduras con poca 
CUADRO 3

Puntajes de las variables para el cálculo del IIBAMA

TABLE 3

Attribute scores of variables used in calculating the IIBAMA

\begin{tabular}{lcccccccc}
\multicolumn{1}{c}{ Sitios } & RT & REPT & RII & \#TI & TM & \#TF & IIBAMA & Categoría IIBAMA \\
RC-04 & 4 & 3 & 4 & 4 & 3 & 4 & 22 & Sub-óptimo \\
RC-05 & 4 & 4 & 4 & 4 & 2 & 4 & 22 & Sub-óptimo \\
RC-06 & 4 & 3 & 4 & 4 & 4 & 3 & 22 & Sub-óptimo \\
RC-07 & 4 & 1 & 4 & 4 & 4 & 2 & 19 & Bueno \\
RC-08 & 2 & 1 & 1 & 2 & 4 & 1 & 11 & Pobre \\
San Miguel & 4 & 4 & 4 & 4 & 4 & 4 & 24 & Óptimo \\
El Campanario & 4 & 4 & 4 & 4 & 4 & 4 & 24 & Óptimo \\
\hline
\end{tabular}

Riqueza de taxa (RT), Riqueza de Ephemeroptera, Plecoptera y Trichoptera (REPT), riqueza de insectos intolerantes (RII), número de taxa intolerantes (\#TI), tolerancia media (TM), número de taxa fijos (\#TF).

pendiente. Sin embargo, aguas abajo el cauce recobra las características del río esperado para el tipo de valle, hasta llegar a los cauces tipo $\mathrm{F}$ asociados al fallamiento de tipo normal que se ubica en la zona (Rosgen, 1996). Las condiciones hidráulicas poco alteradas de la cabecera de la microcuenca le confieren a los sitios localizados en ella las categorías óptimas en cuanto a la calidad de hábitat, presentando poca influencia antropogénica así como en el sitio control que se encuentra cerca del parteaguas. La baja calidad del hábitat en la parte media (sitio RC-08), se debe probablemente a las alteraciones provocadas por la extracción de materiales pétreos y la eliminación de la vegetación de las riberas, lo que permite la entrada lateral de sedimentos finos. De igual modo, las modificaciones de los substratos por actividades ganaderas ubicadas en este sitio, han impactado el estatus del flujo y el gradiente de sedimentación (Barbour et al., 1999). En su conjunto estas afectaciones al cauce, provocan que los substratos se cubran con partículas finas y de este modo se reduce notablemente la complejidad de la estructura de los hábitats. Este proceso disminuye la disponibilidad de substratos para el establecimiento de los macroinvertebrados acuáticos. La recuperación de las condiciones ambientales aguas abajo es notoria ubicando a los sitios en la categoría sub-óptima, recuperando la vegetación ribereña que funciona como trampa de sedimentos y regula la erosión de las riberas (Bentrup, 2008).

En cuanto a la estructura de la comunidad de macroinvertebrados acuáticos, el Campanario y San Miguel son los sitios que mayor similitud tienen y, de acuerdo a las variables del IIBAMA (Pérez \& Pineda, 2005), se ven reflejados los procesos ecológicos que explican la buena integridad biótica de sus comunidades. Donde la alta riqueza de taxa por parte de los insectos tiene un papel clave, así como los grupos Plecoptera, Trichoptera y Ephemeroptera. Una explicación plausible por la que el sitio RC-04 forma parte del grupo de mayor calidad ambiental, es que se observan alteraciones en la calidad de hábitat que aún no han impactado a las riberas, por las que estas aún se encuentran protegidas por la vegetación ribereña y los substratos todavía están disponibles para la epifauna, lo que posiblemente ha permitido que se recupere la riqueza igual a la encontrada en la cabecera sirviendo como zona de resguardo antes de entrar a la ciudad de Morelia, demostrando la capacidad resiliente del cauce (FISRWG, 1998).

En el RC-08 la riqueza de familias de EPT es pobre y además tiene la menor cantidad de taxa fijos, obteniendo las calificaciones más bajas de la microcuenca. La tolerancia media mínima se encuentra asociada a los sitios El Campanario y San Miguel siendo los más 
conservados (Barbour et al., 1999). La tolerancia en el sitio RC-08 es integrada por taxa de tolerancia intermedia, esto es posible probablemente por la buena calidad de sus aguas, lo que permite el establecimiento de biota intolerante en este tramo del cauce el cual está compuesto principalmente por taxa de hábitos nadadores (Segnini, 2003). El sitio RC-07 recibe las descargas de aguas residuales de las poblaciones más grandes de la microcuenca. En contraparte, la comunidad de macroinvertebrados acuáticos ha respondido en su estructura probablemente al incremento de materia orgánica, como lo explica Roldan (2003) y Ríos, Prat \& Encalada (2012), que al incrementarse las descargas se ve afectada la diversidad y aumenta la dominancia, en este caso por la familia Simuliidae que registró el $63 \%$ de la abundancia relativa del sitio y es colector filtrador, encontrando una fuente ideal de alimento para su desarrollo.

En los sitios RC-06 y RC-05 se incrementa la riqueza y abundancia de macroinvertebrados acuáticos, estaciones en que la dinámica hidráulica y las condiciones del hábitat están poco alteradas. Los sitios (RC-05, RC-06 y RC-07) cercanos a la ciudad al estar principalmente estructurados por taxa fijos, con efemerópteros, plecópteros y tricópteros, con riquezas totales mayores a 30, con más de 14 taxa intolerantes entre otras variables, el IIBAMA les confiere el carácter de excelente, pero cercanos a la categoría del sub-óptimo con 22 puntos. La clara correspondencia que se aprecia en el acomodo de los sitios en el análisis de agrupamiento, con la disminución de los valores del VCAV, puede considerarse como evidencia para utilizar a la organización funcional como un estimador de la calidad ambiental.

Para acciones de monitoreo se comprobó que la diversidad, estimada con el índice de Shannon, no necesariamente refleja los efectos producidos por un impacto físico al cauce, corroborando lo expresado por Segnini (2003) y Pérez et al. (2006). Este fenómeno fue ampliamente discutido por Calow (1992), quien encontró que la diversidad no covaría con la calidad ambiental y por lo mismo no se puede considerar como un estimador confiable de la condición de los ecosistemas acuáticos. Mientras que el IIBAMA se encontró altamente correlacionado con la calidad ambiental visual, demostrando que el IIBAMA es una herramienta útil para el monitoreo de la calidad ambiental. Tal como plantea Pérez-Munguía, Torres, Coronado \& Piñon (2008) se observa que tanto la geomorfología el VCAV y el IIBAMA, coinciden detectando a los sitios con mayor o menor problemática, explicando desde diferentes puntos vista los efectos y consecuencias de las acciones que se llevan a cabo en la dinámica hidráulica, y ambiental, viéndose reflejado en la comunidad de macroinvertebrados acuáticos.

En conclusión, la microcuenca del río Chiquito, antes de entrar a la ciudad de Morelia, ha demostrado ser un sistema estable, rico e íntegro, y mantiene los procesos que regulan la dinámica funcional en el cauce donde se observa la capacidad de regresar al estado previo a los impactos, lo que permite el establecimiento de la biota acuática. Las condiciones geomorfológicas que modelan el río están poco impactadas, lo que ha favorecido la conservación de los mecanismos que regulan la dinámica hidráulica de erosión, transporte y sedimentación, sosteniendo la capacidad de autodepuración del río. La integración de la geomorfología, la valoración de la calidad ambiental visual y el índice de integridad biótica para la comunidad de macroinvertebrados acuáticos, ha proporcionado una estrategia que desde diferentes perspectivas permite conocer la condición presente en cada sitio de muestreo, para llevar a cabo acciones de conservación y restauración.

\section{RESUMEN}

La microcuenca del Río Chiquito abastece el 30\% de agua potable para la ciudad de Morelia, Michoacán, México. Por ello es necesario el monitoreo constante de sus aguas, utilizando mediciones que evalúen el deterioro ambiental y la comunidad de macroinvertebrados acuáticos. Para el presente estudio se utilizó el Índice de Integridad Biótica para las Asociaciones de Macroinvertebrados Acuáticos (IIBAMA). Además de la Valoración de la Calidad Ambiental Visual y el estado más probable del cauce, en seis sitios dentro de la microcuenca y un séptimo fuera de la misma, como referencia. Se identificaron un total de 51474 organismos: 69 familias distribuidas en 
cuatro filos, ocho clases, ocho subclases, y 18 órdenes. Los sitios mejor conservados se encuentran en la cabecera de la microcuenca. La zona intermedia se encuentra afectada en la condición hidráulica, calidad ambiental y en su integridad biótica. En la zona baja de la microcuenca, se observó la capacidad "resiliente" del río al restablecerse las comunidades de macroinvertebrados y los parámetros ambientales. Los resultados muestran que la calidad ambiental y el IIBAMA se encuentran correlacionados $(\mathrm{r}=0.82, \mathrm{p}<0.01)$. El uso de los macroinvertebrados en combinación con los parámetros ambientales demuestran que antes de entrar a la ciudad de Morelia la microcuenca del río Chiquito es un sistema estable, diverso e íntegro en su comunidad de macroinvertebrados acuáticos, y que conserva los procesos que regulan la dinámica funcional de la cuenca.

Palabras clave: macroinvertebrados, microcuenca, ensamblajes, integridad biótica, resiliente.

\section{REFERENCIAS}

AFNOR. (1992). Essais des eaux: Détermination de l'indice biologique global normalisé. Norme Francaise, T 90-350.

Alba Tercedor, J., \& Sánchez Ortega, A. (1988). A simple and quick method to evaluate biological quality of running freshwater based on Hellawell (1978). Limnetica, 4, 51-56.

Barbour, M. T., Gerritsen-Zinder, B. D., \& Stribling, J. B. (1999). Rapid Bioassessment Protocols for Use in Streams and Wadeable Rivers: Periphyton, Benthic Macroinvertebrates and Fish. Second Edition. EPA 841-B41-99-002.

Bartram, J., \& Ballance, R. (1996). Water Quality Monitoring: A practical Guide to the Design of Freshwater Quality Studies and Monitoring Programmes. Londres: Chapman Hill.

Bentrup, G. (2008). Zonas de amortiguamiento para conservación: lineamientos para diseño de zonas de amortiguamiento, corredores y vías verdes. Informe Técnico Gral. SRS-109. Asheville, NC: Departamento de Agricultura, Servicio Forestal, Estación Investigación Sur.

Bunte, K., \& Abt, S. R. (2001). Sampling Surface and Subsurface Particle-Size Distributions in Wadable Gravel- and Cobble-Bed Streams for Analyses in Sediment Transport, Hydraulics, and Streambed Monitoring. Gen. Tech. Rep. RMRS-GTR-74. Fort Collins, CO: U.S. Department of Agriculture, Forest Service, Rocky Mountain Research Station.

Calow, P. (1993). Ecosystems not optimized. Journal of Aquatic Ecosystem Health, 2, 55.

Dall, P. C., Friberg, N., Lindegaard, C., \& Toman, M. J. (1995). A practical guide of biological assessment of stream water quality. In M.J. Toman \& F. Steinman
(Eds.), Biological Assessment of stream water quality (pp. 97-145). Ljubljana: University of Ljubljana.

FISRWG. (1998). Stream Corridor Restoration: Principles, Processes and Practices. By the Federal Interagency Stream Restoration Working Group.

Geffrard, M. H., Saulnier-Talbot É., \& Gregory-Eaves, I. (2011). A comparative analysis of fine versus coarse taxonomic resolution in benthic chironomid community analyses. Ecological Indicators, 11, 1541-1551

Gibbson, G. R., Barbour, M. T., Stribling, J. B., Gerritsen, J., \& Karr, J. R. (1996). Biological criteria: Technical guidance for streams and small rivers. Washington: U.S. Environmental Protection Agency, EPA 822-B-96-001.

Honorable Ayuntamiento de Morelia. (2010). Plan de Desarrollo Urbano de Centro de Población de Morelia. Periódico Oficial del Gobierno constitucional del Estado de Michoacán de Ocampo. Recuperado de: http://www.michoacan.gob.mx

INIRENA, Facultad de Biología UMSNH \& Agua Nuestra A. C. (2006). Plan de manejo de la microcuenca del río Chiquito. México: Universidad Michoacana de San Nicolás de Hidalgo.

Karr, J. R., \& Dudley, D. R. (1981). Ecological perspective on water quality goals. Enviromental Management, 5, 55-68.

Kovach Computing Services. (1998). Multivariate Statistical Package (MVSP) v. 3.01.

Lyons, J. (1992). Using the index of biotic integrity (IBI) to measure environmental quality in warmwater streams of Wisconsin. General Technical Report 149, U.S.A. Minnesota: Forest Service, North Central Forest Experiment Station.

Mandaville, S. M. (2002). Benthic macroinvertebrates in freshwaters - Taxa tolerance values, metrics, and protocols. New York: Department of Environmental Conservation.

Merritt, R. W., Cummins, K. W., \& Berg, M. B. (2008). An Introduction to the Aquatic Insects of North America ( $4^{\text {th }}$ Edition). USA: Kendall/Hunt Publishing Company.

Mitchell, L. F., Coan, T. L., McFarland, A. M. S., \& Lasswell, J. L. (1995). Sampling Statistics for Higher Aquatic Taxa. U.S.A: Texas Institute for Applied Environmental Research.

Pennak, R. (1989). Freshwater invertebrates of the United States. Protozoa to Mollusca. (3rd edition). USA: Wiley Interscience.

Pérez, M. R. M., \& Pineda, L. R. (2005). Diseño de un índice de integridad para ríos y arroyos del centro de México, usando las asociaciones de macroinvertebrados. Entomología Mexicana, 4, 241-245. 
Pérez, M. R. M., Arreola, E. P. M., Madrigal, P. R., Ortiz, M. M., Piñón, F. M., Ramírez, A., Ulises, M. V. M., \& Torres, G. U. (2006). Análisis comparativo del índice de integridad biótica con base en las asociaciones de macroinvertebrados acuáticos (IIBAMA) con el índice biológico global normalizado (IBGN) en arroyos y ríos del centro de México. Entomología Mexicana, $5,375-380$

Pérez-Munguía, R. M., Torres, G. U., Coronado, M. N. M., \& Piñón, F. M. A. (2008). Valoración de la calidad ambiental de tres ríos, con distintos impactos, en el estado de Michoacán. $C+T e c$., Edición Especial: 26-31.

Ríos, B., Prat, N., \& Encalada, A. C. (2012). Invertebrate drift and colonization processes in a tropical Andean stream. Aquatic Biology, 14, 233-246.

Roldan, G. A. (2003). Bioindicación de la calidad del agua en Colombia, uso del método BMWP/Col. Colombia: Impresa Universidad de Antioquia.

Rosgen, D. (1996). Applied river morphology. USA: Wilde land Hydrology.
SAS. (2008). JMP v.8. USA: Statistical Discovery from SAS Institute.

Segnini, S. (2003). El uso de los macroinvertebrados bentónicos como indicadores de la condición ecológica de los cuerpos de agua corriente. Sociedad Venezolana de Ecología. Ecotropicos, 16, 45-63.

Székely, G. J., \& Rizzo, M. L. (2005). Hierarchical Clustering via Joint Between-Within Distances: Extending Ward's Minimum Variance Method. Journal of Classification, 22, 151-183.

Thorp, J. H., \& Covich, A. P. (2001). Ecology and classification of North American freshwater invertebrates (2nd Edition). USA: Academic Press.

Wiggins, G. (1994). Larvae of the North American caddisfly genera (Trichoptera). (2nd Edition). Canada: University of Toronto Press.

Williams, P. H., \& Gaston, K. J. (1994). Measuring more of biodiversity: Can higher taxon richness predict who sale species richness? Biological Conservation, 67, 211-217. 
